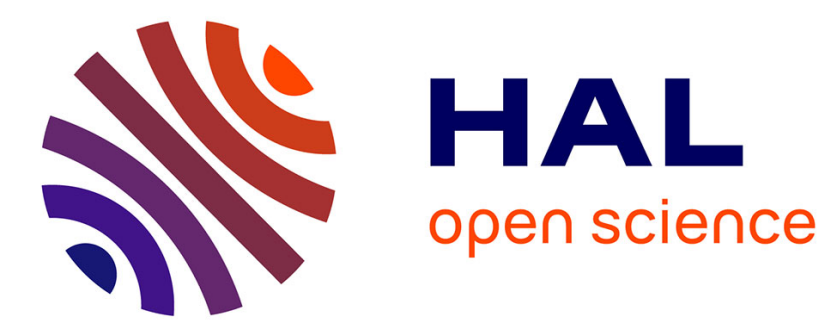

\title{
Theory of spin dynamics in high-Tc superconductors
}

\author{
M. Lavagna, G. Stemmann, C. Pépin
}

\section{To cite this version:}

M. Lavagna, G. Stemmann, C. Pépin. Theory of spin dynamics in high-Tc superconductors. Physica

B: Condensed Matter, 1995, 215 (1), pp.62 - 70. 10.1016/0921-4526(95)00026-6 . hal-01896231

\section{HAL Id: hal-01896231 \\ https://hal.science/hal-01896231}

Submitted on 17 Oct 2018

HAL is a multi-disciplinary open access archive for the deposit and dissemination of scientific research documents, whether they are published or not. The documents may come from teaching and research institutions in France or abroad, or from public or private research centers.
L'archive ouverte pluridisciplinaire HAL, est destinée au dépôt et à la diffusion de documents scientifiques de niveau recherche, publiés ou non, émanant des établissements d'enseignement et de recherche français ou étrangers, des laboratoires publics ou privés. 


\title{
Theory of spin dynamics in high- $T_{\mathrm{c}}$ superconductors
}

\author{
M. Lavagna*, G. Stemmann, C. Pépin \\ Centre d'Etudes Nucléaires de Grenoble, Département de Recherche Fondamentale sur la Matière Condensée, SPSMS, \\ 17 Rue des Martyrs, 38054 Grenoble Cedex 9, France
}

Received 7 December 1994

\begin{abstract}
We analyze the spectrum of magnetic excitations as observed by neutron diffraction and NMR experiments in $\mathrm{YBa}_{2} \mathrm{Cu}_{3} \mathrm{O}_{6+x}$, in the frame of the single-band $t-t^{\prime}-J$ model in which the next-nearest neighbor hopping term has been introduced in order to fit the shape of the Fermi surface revealed in photoemission. Within the slave-boson approach, we have as well examined the $d$-wave superconducting state, and the singlet-RVB phase appropriate to describe the normal state of heavily doped systems. Our calculations show a smooth evolution of the spectrum from one phase to the other, with the existence of a spin-gap in the frequency dependence of $\chi^{\prime \prime}(\boldsymbol{Q}, \omega)$. The value of the threshold of excitations $E_{\mathrm{G}}$ is found to increase with doping, while the characteristic temperature scale $T_{\mathrm{m}}$ at which the spin-gap opens, exhibits a regular decrease, reaching $T_{\mathrm{c}}$ only in the overdoped regime. This very typical combined variation of $E_{\mathrm{G}}$ and $T_{\mathrm{m}}$ with doping results of strong-correlation has its effect in the presence of a realistic band structure. We point out the presence of a resonance in the $\omega$-dependence of $\chi^{\prime \prime}(\boldsymbol{Q}, \omega)$ in good agreement with the neutron diffraction results obtained at $x=0.92$ and $x=1.0$. This resonance is interpreted as a dynamical Kohn anomaly of the second kind in the Cooper channel. Finally, we examine the $q$-dependence of the dynamical susceptibility allowing to study the magnetic correlation length $\xi$ as a function of doping, frequency and temperature.
\end{abstract}

\section{Introduction}

In this paper, we address the problem of the theoretical understanding of the spin-excitations in high- $T_{\mathrm{c}}$ superconductors. Inelastic neutron scattering (INS) and nuclear magnetic resonance (NMR) experiments performed in various cuprates, both in the normal and in the superconducting states, rise a number of fundamental questions that are very important to answer. Let us here summarize the essential features revealed in INS and NMR:

1. In the metallic phase above a critical value of doping, long-range antiferromagnetic order disappears but the dynamical spin structure factor $S(q, \omega)$ keeps large amplitudes centered around the antiferromagnetic vector

\footnotetext{
* Corresponding author. Member of the Centre National de la Recherche Scientifique (CNRS).
}

$\boldsymbol{Q}=(\pi, \pi)$ in $\mathrm{YBa}_{2} \mathrm{Cu}_{3} \mathrm{O}_{6+x}$ compounds [1-13], or $q=(\pi \pm \delta q, \pi)$ and $q=(\pi, \pi \pm \delta q)$ in $\mathrm{La}_{2-x} \mathrm{Sr}_{x} \mathrm{CuO}_{4}$ compounds [14] signalling commensurate or incommensurate spin-fluctuations, respectively.

2. The magnetic correlation length $\xi$ deduced from the width of the $q$-dependence of $S(q, \omega)$ around its maximum decreases regularly with doping. The fact that $\xi$ does not exhibit any significant dependence on temperature and frequency (except in the vicinity of the resonance outlined below) is undoubtedly an important point that has been thoroughly examined in the course of our work.

3. Valuable information can be extracted from the frequency dependence of $\boldsymbol{S}(\boldsymbol{Q}, \omega)$ at low temperatures. In yttrium compounds, a depression of intensity is observed at low frequencies with a finite cut-off of the order of $50 \mathrm{meV}$. The question concerning the existence of a gap in the spectrum of spin-excitations $S(Q, \omega)$ is presently extensively discussed. Rossat-Mignod et al. [9] report the 
formation of a gap, the value of which continuously varies with doping: $E_{\mathrm{G}}=4,16,28$ and $26 \mathrm{meV}$ at, respectively, $x=0.51,0.69,0.92$ and 1.0 . The corresponding superconducting temperature $T_{\mathrm{c}}$ of $47,60,91$ and $89 \mathrm{~K}$ exhibits a maximum at a critical concentration. This behavior is at the origin of the terminology introduced, with the distinction between heavily doped (here $x=0.51,0.69$ and 0.92 ) and overdoped systems (here $x=1.0$ ). The difference between these two regimes is also reflected in the temperature scale $T_{\mathrm{m}}$ at which the gap opens. Experimentally, $T_{\mathrm{m}}$ is determined from the position of the maximum of $S\left(Q, \omega_{0}\right)\left(\omega \ll E_{\mathrm{G}}\right)$ with temperature. $T_{\mathrm{m}}$ is larger than $T_{\mathrm{c}}$ in heavily doped systems, from which originates the appellation of a pseudo-gap or a spin-gap in this case. Conversely, the gap disappears immediately at $T_{\mathrm{c}}\left(T_{\mathrm{m}}=T_{\mathrm{c}}\right)$ in the overdoped regime. The corresponding results are shown in the schematic phase diagram of Fig. 1(a). The fact that in this series of experiments, $T_{m}$ decreases with doping while $E_{\mathrm{G}}$ increases may be surprising at first sight. Moreover, $E_{\mathrm{G}}$ is found to be rather small compared to the superconducting gap reaching a value of only $3.5 T_{\mathrm{c}}$ in the overdoped regime. These last two points have been thoroughly discussed in the framework of the strong-coupling regime considered in our work, Generally, a large controversy still exists on the existence of the spin-gap. The measurements are in fact particularly delicate due to the smallness of the signal itself, especially at high doping when $\Delta q$ becomes large, and also due to the difficulty in extracting the magnetic contribution. Another experiment [6] realized at $x=0.6$ leads to a gap properly interpolating between the values reported by Rossat-Mignod et al. at the neighboring concentrations $x=0.51$ and 0.69 . However, other studies [2] performed at different concentrations have led so far to negative answers as regards the formation of the spin-gap, in spite of the natural arguments in favor of its existence coming from the lowtemperature behavior of the nuclear relaxation time on ${ }^{63} \mathrm{Cu}$ measured by NMR (cf. below). Whatever the final issue concerning the experimental controversy about the existence of the spin-gap, it appears crucial on the theoretical side to examine the question without any a priori assumptions on its existence taking into account all the indications coming from the experiments, so far instance the shape of the Fermi surface determined by angleresolved photoemission experiments and confirmed by band structure calculations.

4. The presence of a resonance at $41 \mathrm{meV}$ in $S(Q, \omega)$ is now well established for highly doped $\mathrm{YBa}_{2} \mathrm{Cu}_{3} \mathrm{O}_{6+x}$ systems. This resonance was first reported by RossatMignod [1,9] for the concentrations $x=0.92$ and 1.0 with a characteristic enhancement of the magnetic correlation length $\xi$ in its vicinity. It has recently been confirmed for the later composition using polarized

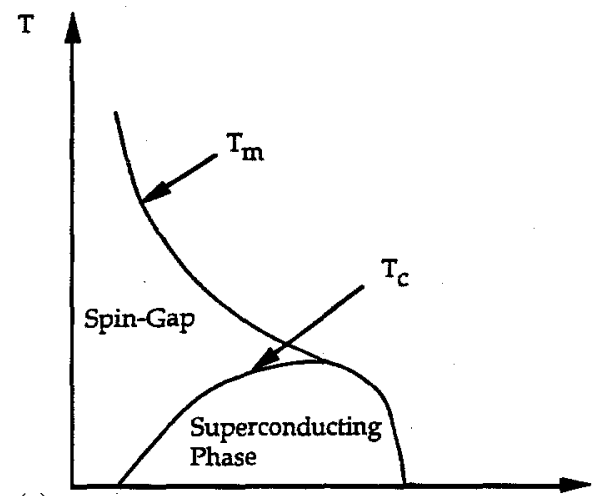

(a)

(b)

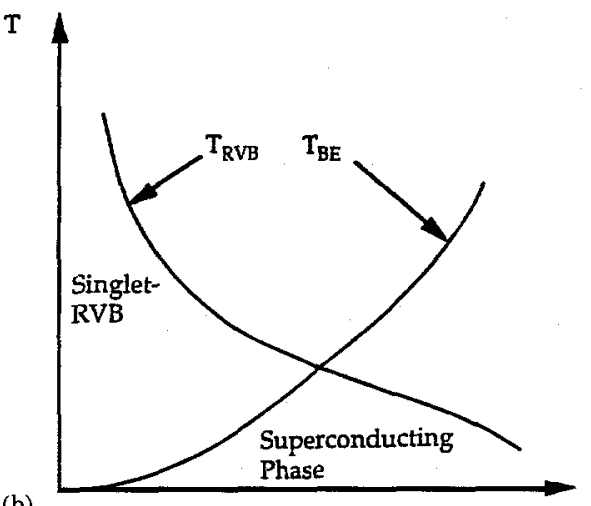

Fig. 1. Schematic phase diagrams for $\mathrm{YBa}_{2} \mathrm{Cu}_{3} \mathrm{O}_{6+x}$ : (a) sketch of experimental results; (b) sketch of theoretical results within the RVB hypothesis.

neutron scattering techniques [10]. The existence of this resonance has not yet received any interpretation, and it is one of the purposes of our work to answer this question.

5. The measurements of the nuclear relaxation rate [15-18] on ${ }^{63} \mathrm{Cu}$ are consistent with the opening of a spin-gap in the spectrum of antiferromagnetic fluctuations. ${ }^{63}\left(T_{1} T\right)^{-1}$ is large and does not exhibit a Korringa law but has instead a maximum as a function of temperature at the same temperature $T_{\mathrm{m}}$ as seen in INS. This is expected since the hyperfine constant of ${ }^{63} \mathrm{Cu}$ mostly filters the $Q=(\pi, \pi)$ component. On the other hand, the nuclear relaxation rates on ${ }^{89} \mathrm{Y}$ and ${ }^{17} \mathrm{O}$ involving different $q$ filtering have a different temperature behavior [19-21]. Both these quantities show a decrease when lowering the temperature, starting from well above $T_{\mathrm{c}}$ in heavily doped systems. Once more, the overdoped case is special since the decrease starts directly at $T_{\mathrm{c}}$. In 
a non-conventional way, $\left.\left(T_{1} T\right)\right)^{-1}$ on ${ }^{89} \mathrm{Y}$ and ${ }^{17} \mathrm{O}$ varies linearly with the Knight shift.

The whole set of the INS and NMR data constitutes a puzzling problem in that the spin-excitation spectrum drastically differs from a traditional Fermi liquid. We want to mention that a number of theories have been developed these last years to understand this class of behavior, starting from rather different points of view: quantum disordered description of the normal phase [22], marginal Fermi liquid [23], or more microscopic approaches starting from an electronic description of the $\mathrm{CuO}_{2}$ layers. It is widely accepted that the high $T_{\mathrm{c}}-$ cuprates come under the strong coupling regime reached either in multi-band [24-26] or single-band descriptions [27-31] formed from the Zhang and Rice [32] singlets $\mathrm{Cu}-\mathrm{O}$ in the layers. The role of the next-nearest hopping term $t^{\prime}$ is then crucial in order to reproduce a Fermiology in agreement with the angle-resolved photoemission results [33-36] on the shape of the Fermi surface. As has been pointed out in previous work for the weak coupling limit $\left(t-t^{\prime}-U\right.$ Hubbard model), the effect of $t^{\prime}\left(t^{\prime}<0\right)$ makes the model a better starting point from a perturbative point of view:

(a) The Fermi surface is found to be rotated $45^{\circ}$ compared to the diamond shape and centered around the point $S(\pi, \pi)$ instead of $\Gamma(0,0)$.

(b) It leads to the right sign of the Hall effect (cf. curvature of the Fermi surface) and the correct doping dependence of the Knight shift [37,38].

(c) As concerns the spin-excitations, the prediction for the weak-coupling regime $[39,40]$ is the absence of a gap in the frequency dependence of $\chi^{\prime \prime}(Q, \omega)$ for the current regime of interest $\left(4 t^{\prime}<\mu<0\right)$, as long as pairing effects are not considered ( $4 t^{\prime}$ locates the position of the Van Hove singularity in the density of states).

On the other hand, when pairing effects are introduced, with for instance a d-wave symmetry of the order parameter, it has been shown [40]. that $\chi^{\prime \prime}(\boldsymbol{Q}, \omega)$ develops a gap with a very characteristic evolution of the threshold of excitations $E_{G}$ with doping. In addition to the gap, the model leads to the prediction of a resonance in clear analogy with the experimental results obtained in $\mathrm{YBa}_{2} \mathrm{Cu}_{3} \mathrm{O}_{6+x}$ at $T<T_{\mathrm{c}}$. The resonance has been interpreted as a dynamical Kohn anomaly of the second kind in the Cooper channel and is typical of axial superconductivity.

Motivated by the striking resemblance between the calculated spectrum of magnetic excitations in the superconducting state of the weak-coupling regime, and the spectrum observed in the normal phase of heavily doped $\mathrm{YBa}_{2} \mathrm{Cu}_{3} \mathrm{O}_{6+x}$, we examine in this paper the strongcoupling limit expressed in the $t-t^{\prime}-J$ model with the idea of extending the pairing effects to the singlet resonant-valence-bond (RVB) phase above $T_{\mathrm{c}}$. This problem, already addressed in some recent work [41-43], will be considered here [44-46] closer to the systematics that we developed earlier in the weak-coupling limit. Similar studies have recently been performed by other authors $[47,48]$ using diagrammatic techniques for Hubbard operators.

\section{Mean-field slave-boson approach of the $t-t^{\prime}-J$ model}

The low-lying excitations in the $\mathrm{CuO}_{2}$ planes are believed to be described by a generalized $t-t^{\prime}-J$ model in which $J$ is the Anderson superexchange coupling between neighboring $\mathrm{Cu}$ spins, $t, t^{\prime}$ represent, respectively, the nearest-neighbor and next-nearest-neighbor transfer integrals of the Zhang and Rice singlets constituted after doping by both $\mathrm{Cu}$ and $\mathrm{O}$ spins. In the slave-boson representation, the $t-t^{\prime}-J$ Hamiltonian is written as

$$
H=-t \sum_{\langle i, j\rangle} c_{i \sigma}^{\dagger} c_{j \sigma} e_{i} e_{j}^{\dagger}-t^{\prime} \sum_{\langle i, j\rangle^{\prime}} c_{i \sigma}^{\dagger} c_{j \sigma} e_{i} e_{j}^{\dagger}+J \sum_{\langle i, j\rangle} S_{i} \cdot S_{j},
$$

in which the spin is expressed in a fermionic representation, $S_{i}$ is noted as $S_{i}^{\alpha}=\sum_{\sigma \sigma^{\prime}} c_{i \sigma}^{\dagger} \tau^{\alpha} c_{i \sigma^{\prime}}$. As usual, the local constraint

$e_{i}^{\dagger} e_{i}+\sum_{\sigma} c_{i \sigma}^{\dagger} c_{i \sigma}=1$

is enforced at each site by the time-independent Lagrange multipliers $\lambda_{i}$. Using the Feynman variational principle, one can find an upper bound to the free energy according to $F \leqslant F_{0}+\left\langle H-H_{0}\right\rangle_{H_{0}}$ in which $\left\langle H-H_{0}\right\rangle_{H_{0}}$ is the average performed on the trial Hamiltonian $H_{0}$ taken as

$$
\begin{aligned}
H_{0}= & -t \sum_{\langle i, j\rangle} F_{i j} c_{i \sigma}^{\dagger} c_{j \sigma}-t^{\prime} \sum_{\langle i, j\rangle} F_{i j}^{\prime} c_{i \sigma}^{\dagger} c_{j \sigma} \\
& -t \sum_{\langle i, j\rangle} B_{i j} e_{i}^{\dagger} e_{j}-t^{\prime} \sum_{\langle i, j\rangle^{\prime}} B_{i j}^{\prime} e_{i}^{\dagger} e_{j} \\
& +\left[\sum_{\langle i, j\rangle} \Delta_{i j}\left(c_{i \uparrow}^{\dagger} c_{j \downarrow}^{\dagger}-c_{i \downarrow}^{\dagger} c_{j \dagger}^{\dagger}\right)+\text { h.c. }\right] \\
& +\sum_{i} \lambda_{i}\left[e_{i}^{\dagger} e_{i}+\sum_{\sigma} c_{i \sigma}^{\dagger} c_{i \sigma}-1\right] \\
& -\mu\left[\sum_{i \sigma} c_{i \sigma}^{\dagger} c_{i \sigma}-N(1-\delta)\right] .
\end{aligned}
$$

The free energy $F_{0}$ can be calculated from $F_{0}=-k_{\mathrm{B}} T \ln Z_{0}$ in which the partition function $F_{0}$ is 
expressed as a functional integral over coherent states of Fermi and Bose fields,

$$
\begin{aligned}
Z_{0}= & \int \mathrm{d} \lambda_{i} D c_{\sigma} D e_{i} \\
& \times \exp \left[-\int_{0}^{\beta} \mathrm{d} \tau\left[\sum_{i \sigma} c_{i \sigma}^{\dagger} \partial_{\tau} c_{i \sigma}+e_{i}^{\dagger} \partial_{\tau} e_{i}+H_{0}\right]\right]
\end{aligned}
$$

At the mean-field level, the bond variables $F_{i, j}^{\left({ }^{\prime}\right)}$ and $B_{i, j}^{\left({ }^{\prime}\right)}$ are considered real and both time and bond independent (s-wave symmetry), while $\Delta_{i, j}=\Delta_{0}(-1)^{\eta}$, with $\eta=2$ (or 1) depending on whether horizontal (or vertical) bonds are involved $\left(d_{x^{2}-y^{2}}\right.$ symmetry) $\gamma_{k}=\left(\cos k_{x}+\right.$ $\left.\cos k_{y}\right) / 2$ for $\mathrm{NN}\left(\gamma_{k}^{\prime}=\cos k_{x} \cos k_{y}\right.$ for $\left.\mathrm{NNN}\right)$ and $\alpha_{k}=\left(\cos k_{x}-\cos k_{y}\right) / 2$ are, respectively, associated to these two types of symmetries. $\lambda_{i}$ is supposed to be site independent. The saddle-point equations are

$F=\frac{1}{N} \sum_{k} \gamma_{k} n_{\mathrm{B}}\left(\bar{\eta}_{k}\right)+\frac{3 J}{8 t} B$,

$F^{\prime}=\frac{1}{N} \sum_{k} \gamma_{k}^{\prime} n_{\mathrm{B}}\left(\bar{\eta}_{k}\right)$,

$B^{\left({ }^{\prime}\right)}=\frac{-2}{N} \sum_{k} \gamma_{k}^{\left.\prime^{\prime}\right)} \frac{\bar{\xi}_{k}}{2 \varepsilon_{k}} \tanh \left(\beta \varepsilon_{k} / 2\right)$,

$1=3 J \frac{1}{N} \sum_{k} \alpha_{k}^{2} \frac{1}{2 \varepsilon_{k}} \tanh \left(\beta \varepsilon_{k} / 2\right)$,

$1-\delta=\frac{1}{N} \sum_{k}\left[1-\frac{\bar{\xi}_{k}}{2 \varepsilon_{k}}\right] \tanh \left(\beta \varepsilon_{k} / 2\right)$,

$\delta=\frac{1}{N} \sum_{k} n_{\mathrm{B}}\left(\bar{\eta}_{k}\right)$

in which

$$
\begin{aligned}
& n_{\mathrm{B}}(E)=\frac{1}{\exp (\beta E)-1}, \\
& n_{\mathbf{F}}(E)=\frac{1}{\exp (\beta E)+1}, \\
& \varepsilon_{k}=\sqrt{\xi_{k}^{2}+\Delta_{k}^{2}}, \\
& \xi_{k}=\xi_{k}+\lambda-\mu, \\
& \xi_{k}=-z \gamma_{k} t F-z \gamma_{k}^{\prime} t^{\prime} F^{\prime}, \\
& \Delta_{k}=z \alpha_{k} \Delta_{0}, \\
& \bar{\eta}_{k}=\eta_{k}-\lambda, \\
& \eta_{k}=-z \gamma_{k} t B-z \gamma_{k}^{\prime} t^{\prime} B^{\prime} .
\end{aligned}
$$

Eqs. (4a) (4c) give the renormalization of boson and fermion electronic transfer integrals resulting from strong-correlation effects. Eq. (4d) is the equivalent of the gap equation of the BCS theory in its strong-coupling version since the coefficient $\alpha_{k}^{2}$ reflects the formation of Cooper pairs along nearest-neighbor bonds. Eq. (4e) expresses the conservation of the average number of particles, and Eq. (4f) corresponds to the constraint that is only globally fulfilled at the mean-field level.

Two characteristic temperatures arise from the meanfield approximation as schematically illustrated in the phase diagram reported in Fig. 1(b): the Bose condensation temperature of holons $T_{\mathrm{BE}}$ (defined by $\mu_{\mathrm{B}}\left(T_{\mathrm{BE}}\right)=0$ ), and the pairing temperature of spinons $T_{\mathrm{RVB}}$ (defined by $\left\langle c_{i \uparrow}^{\dagger} c_{j \uparrow}^{\dagger}-c_{i \downarrow}^{\dagger} c_{j \downarrow}^{\dagger}\right\rangle \neq 0$ ). This type of theoretical diagram originally proposed by Rice [49] following the ideas of Anderson [50] has been extensively used in the literature $[51,52,41]$. Note that strictly speaking. $T_{\mathrm{BE}}$ should be zero whatever the doping is resulting from general arguments on Bose condensation in two dimensions. This is no longer true if one allows for additional coupling between layers with a variation of $T_{\mathrm{BE}}$ as a function of doping as represented in Fig. 1(b). The superconducting state is obtained when simultaneously spinons are paired and holons condensed, such that $T_{\mathrm{c}}$ is given by $T_{\mathrm{BE}}$ below $\delta_{\mathrm{c}}$, by $T_{\mathrm{RVB}}$ above $\delta_{\mathrm{c}}$, and then exhibits the non-monotonic behavior quoted earlier. The RVB state in the slaveboson representation corresponds to $T_{\mathrm{BE}}<T<T_{\mathrm{RVB}}$, for which pairing of spinons does not transpose into pairing of physical particles, since the condensate of holons has lost its macroscopic occupation. In the case of the $t-t^{\prime}-J$ model within its slave-boson representation, we have numerically solved the saddle-point equations and reported the results in Figs. 2 and 3. A considerable simplification occurs at low temperatures, when the bosons are either partially condensed $\left(T<T_{\mathrm{BE}}\right)$, or occupy the first excited levels $\left(T>T_{\mathrm{BE}}\right)$ with $\gamma_{k}^{(\prime)}=1$. In both cases, $(1 / N) \sum_{k} \gamma_{k}^{(')} n_{\mathrm{B}}\left(\eta_{k}\right)$ equals $\delta$. When simultaneously $\Delta_{0}$ is finite, the first case corresponds to the

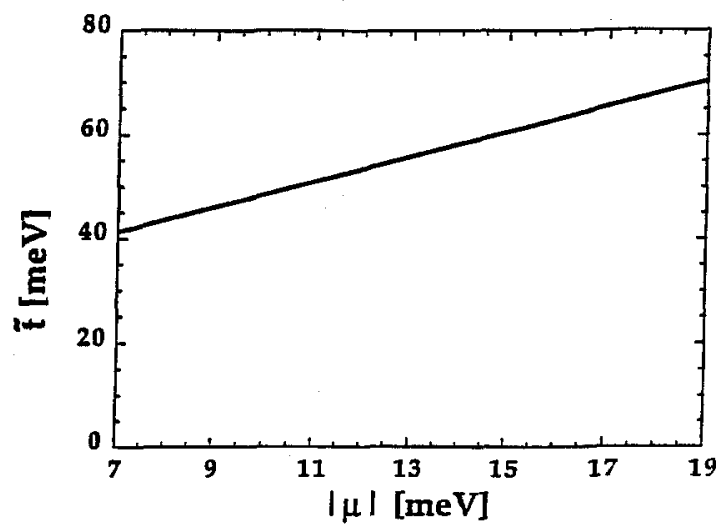

Fig. 2. Effecive nearest-nerighbor transfer integral versus chemical potential. 

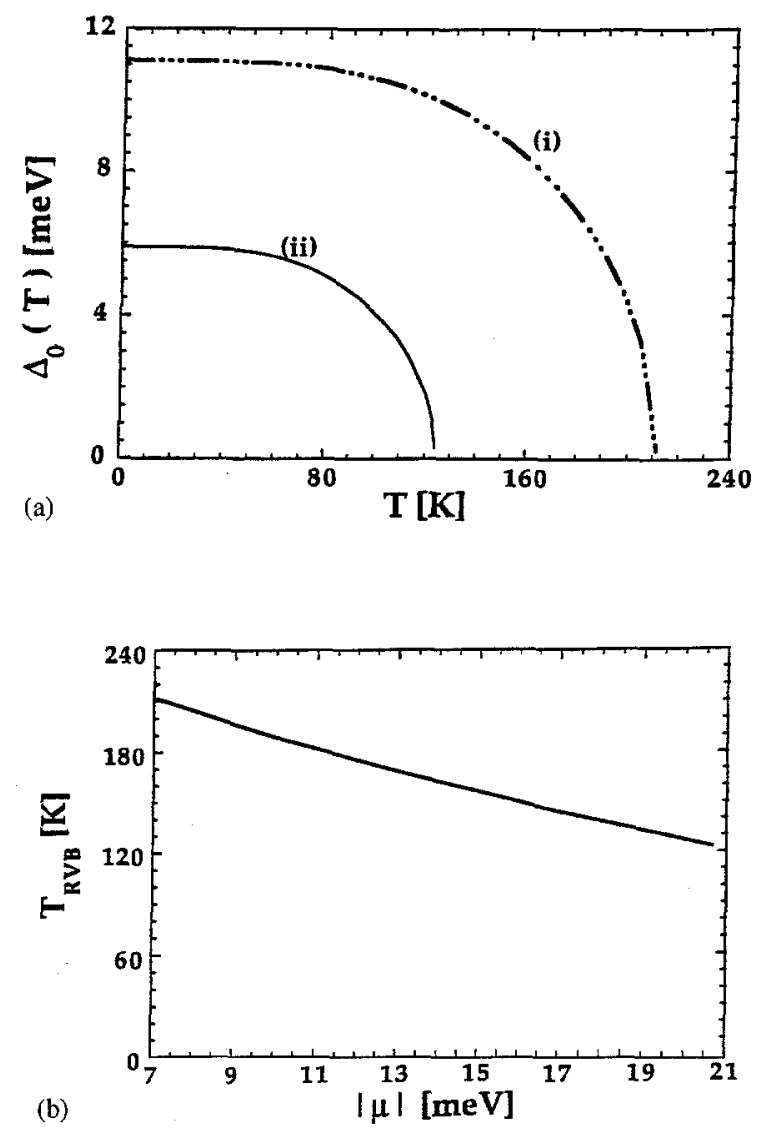

Fig. 3. (a) Pairing parameter $\Delta_{0}$ as a function of temperature obtained for doping concentrations corresponding to heavily doped and overdoped systems, respectively. The corresponding set of parameters are: (i) $\tilde{t}=62 \mathrm{meV}, \tilde{t}^{\prime}=-0.8 \tilde{t}, \mu=$ $-7.05 \mathrm{meV}, \quad \Delta_{0}=13.19 \mathrm{meV}$ (heavily doped) and (ii) $\tilde{t}=74 \mathrm{meV}, \tilde{t}^{\prime}=-0.8 \tilde{t}, \mu=-20.63 \mathrm{meV}, \Delta_{0}=5.64 \mathrm{meV}$ (overdoped). (b) Temperature $T_{\mathrm{RVB}}$ versus chemical potential.

superconducting state, and the second to the singlet-RVB state according to the terminology introduced above. Let us make two comments at this point.

First, the brute-force resolution of the saddle-point equations leads to a sharp decrease of the renormalization parameters $F$ and $F^{\prime}$ when doping is lowered. This feature reflects the underlying Brinkman-Rice transition which occurs as the doping goes to zero. As outlined in earlier work $[37,38]$, this transition has unphysical effects with a sharp increase of the susceptibility at low doping and huge values of the effective masses, which seem not to be observed experimentally. One way out already suggested in Ref. [38] is to push the approach of the Brinkman-Rice transition to very low doping as represented in Fig. 2 (cf. $\tilde{t}=t F$ versus $\mu$ ).
The second comment has to do with the validity of the rigid-band model in which the different parameters $t, t^{\prime}$ and $J$ are kept fixed, whatever the doping. A realistic description of the cuprate superrconductors would require the consideration of more general multi-band models to account for the different electronic states. In this scheme, the determination of the low-lying excitations allows the mapping of the model into effective single-band models, the parameters of which can be systematically determined. Recent work [53] has shown a significant linear decrease of the effective superexchange coupling $J$ with doping. Our calculation take account of this effect, assuming a linear decrease of $J / t$ from 0.23 to 0.20 when $\mu$ evolves from the heavily doped to the overdoped regime. Given that, the results coming from the numerical resolution of the saddle-point equations are reported in Fig. 3(a) for the temperature dependence of $\Delta_{0}$ at various doping, and in Fig. 3(b) for the doping dependence of $T_{\mathrm{RVB}}$. The results are in agreement with the heuristic phase diagram discussed above. Note that our numerical results give $\Delta_{0}=2.3 T_{\mathrm{RVB}}$ with only small doping dependent corrections. This value is to be compared to $\Delta_{0}=1.7 T_{\mathrm{c}}$ of the weak-coupling BCS theory, which essentially results from the effects of $\alpha_{k}^{2}$ in the gap equation (formation of Cooper pairs on bonds).

\section{Spin dynamics}

The dynamical form factor $S(q, \omega)$ is related to the imaginary part of the dynamical susceptibility through the fluctuation-dissipation theorem

$S(\boldsymbol{q}, \omega)=\frac{1}{\pi} \frac{1}{1-\exp (-\beta \omega)} \chi^{\prime \prime}(\boldsymbol{q}, \omega)$.

The dynamical susceptibility is given by the analytical continuation $\mathrm{i} \omega_{n} \rightarrow \omega+\mathrm{i} \delta$ of $\chi\left(\boldsymbol{q}, \mathrm{i} \omega_{n}\right)$ defined in terms of the correlation functions of the physical particles,

$$
\begin{aligned}
\chi\left(\boldsymbol{q}, \mathrm{i} \omega_{n}\right)= & 2 \int \frac{\mathrm{d}^{2} r}{(2 \pi)^{2}} \int \mathrm{d} r \exp \left[\mathrm{i}\left(\omega_{n} \tau-\boldsymbol{q} \cdot \boldsymbol{r}\right)\right] \\
& \times\left\langle T_{\tau}\left[f_{r \sigma}^{\dagger}(\tau) f_{r \bar{\sigma}}(\tau) f_{0 \sigma}^{\dagger}(0) f_{0 \sigma}(0)\right]\right\rangle .
\end{aligned}
$$

In the slave-boson representation, a straightforward calculation (benefiting from the constraints) gives that the correlation function of physical particles reduces to that of only spinons:

$$
\begin{aligned}
& \left\langle T_{\tau}\left[f_{r \sigma}^{\dagger}(\tau) f_{r \bar{\sigma}}(\tau) f_{0 \bar{\sigma}}^{\dagger}(0) f_{0 \sigma}(0)\right]\right\rangle \\
& \quad=\left\langle T_{\tau}\left[c_{r \sigma}^{\dagger}(\tau) c_{r \bar{\sigma}}(\tau) c_{0 \bar{\sigma}}^{\dagger}(0) c_{0 \sigma}(0)\right]\right\rangle .
\end{aligned}
$$




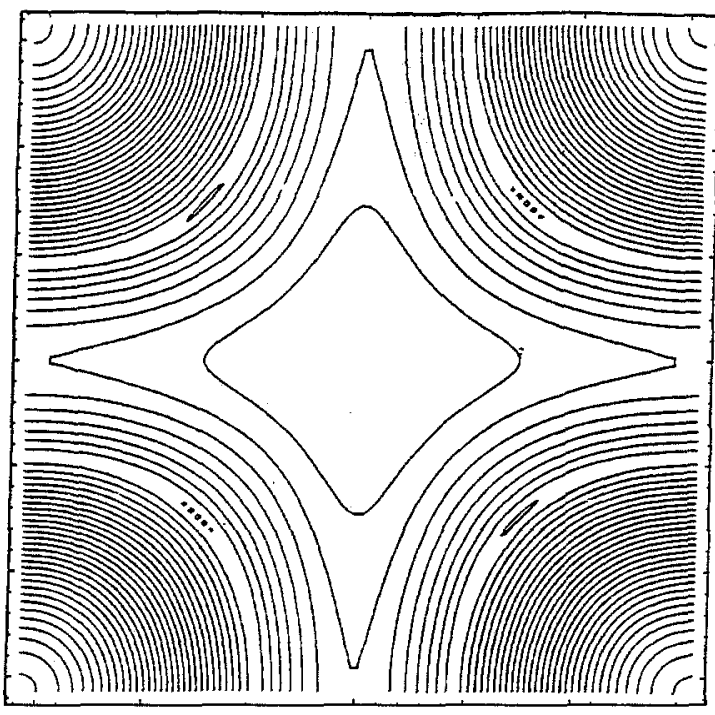

(a)

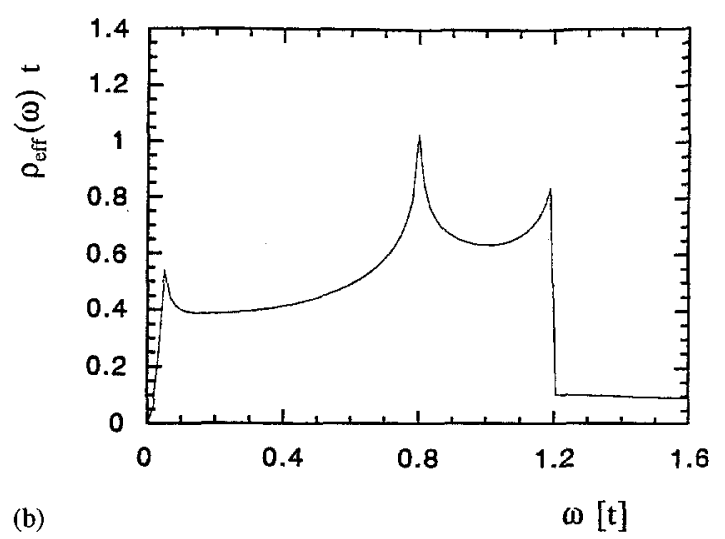

Fig. 4. (a) Equienergetics $\varepsilon_{k}=c^{\text {ste }}$ in the presence of d-wave pairing. Note the formation of four superconducting ellipses around the nodes $\varepsilon_{k}=0$. The parameters choosen are $\tilde{t}=75 \mathrm{meV}, \tilde{t}^{\prime}=-0.45 t, \mu=-125 \mathrm{meV}, \Delta_{0}=3.75 \mathrm{meV}$. (b) Density of states of individual excitations versus energy for the same set of parameters as in (a). Note the linear behavior at small frequencies due to the contribution of superconducting ellipses reflecting the d-wave symmetry of the order parameter $\Delta_{k}$.

In the random-phase approximation, the dynamical susceptibility is then given by

$\chi\left(\boldsymbol{q}, \mathrm{i} \omega_{n}\right)=\frac{\chi_{0}\left(\boldsymbol{q}, \mathrm{i} \omega_{n}\right)}{1+2 J_{\gamma_{q} \chi_{0}}\left(\boldsymbol{q}, \mathrm{i} \omega_{n}\right)}$ in which $\chi_{0}\left(q, i \omega_{n}\right)$ is the bare susceptibility involving both normal and anomalous contributions as usual in the BCS theory of superconductivity according to

$$
\begin{aligned}
\chi_{0}\left(q, \mathrm{i} \omega_{n}\right)= & \sum_{k} \frac{1}{4}\left[1+\frac{\bar{\xi}_{k+q}}{\varepsilon_{k+q}}-\frac{\bar{\xi}_{k}}{\varepsilon_{k}}-\frac{\bar{\xi}_{k} \bar{\xi}_{k+q}+\Delta_{k} \Delta_{k+q}}{\varepsilon_{k} \varepsilon_{k+q}}\right] \\
& \times \frac{n_{\mathrm{F}}\left(\varepsilon_{k}\right)+n_{\mathrm{F}}\left(\varepsilon_{k+q}\right)-1}{\mathrm{i} \omega_{n}-\varepsilon_{k+q}-\varepsilon_{k}} \\
& +\sum_{k} \frac{1}{4}\left[1-\frac{\bar{\xi}_{k+q}}{\varepsilon_{k+q}}+\frac{\bar{\xi}_{k}}{\varepsilon_{k}}-\frac{\bar{\xi}_{k} \xi_{k+q}+\Delta_{k} \Delta_{k+q}}{\varepsilon_{k} \varepsilon_{k+q}}\right] \\
& \times \frac{1-n_{\mathrm{F}}\left(\varepsilon_{k}\right)-n_{\mathrm{F}}\left(\varepsilon_{k+q}\right)}{\mathrm{i} \omega_{n}+\varepsilon_{k+q}+\varepsilon_{k}} \\
& +\sum_{k} \frac{1}{2}\left[1+\frac{\xi_{k} \bar{\xi}_{k+q}+\Delta_{k} \Delta_{k+q}}{\varepsilon_{k} \varepsilon_{k+q}}\right] \\
& \times \frac{n_{\mathrm{F}}\left(\varepsilon_{k+q}\right)+n_{\mathrm{F}}\left(\varepsilon_{k}\right)}{\mathrm{i} \omega_{n}-\varepsilon_{k+q}+\varepsilon_{k}} .
\end{aligned}
$$

We have sketched in Fig. 4(a) the spectrum of individual excitations $\varepsilon_{k}=c^{\text {ste }}$ involved. Let us note the formation of four ellipses around nodes $\left(\varepsilon_{k}=0\right)$ at the intersection of the Fermi surface and of the first bisectors along which the gap vanishes. This feature is characteristic of an axial pairing $\left(\mathrm{d}_{x^{2}-y^{2}}\right.$ symmetry in the present case) in which the gap parameter vanishes at points of the Fermi surface. Far from the Fermi surface, one notes the presence of closed and open orbits centered around the points $\Gamma(0,0)$ and $S(\pi, \pi)$ of the Brillouin zone, respectively. The corresponding density of excitations is represented in Fig. 4(b) with a very characteristic triple-peak structure. The low-energy part coming from ellipse contribution is linear as expected from topological arguments in the case of axial pairing in 2D.

At zero temperature, we have reported in Fig. 5 the $\omega$-dependence of $\chi^{\prime \prime}(\boldsymbol{Q}, \omega)$ that we obtained for two different values of doping corresponding to under-doped and overdoped cases. Note in the latter case the presence of a gap value $E_{\mathrm{G}}=37 \mathrm{meV}$ followed by a resonance at $\omega_{\mathbf{R}}=47 \mathrm{meV}\left(T_{\mathbf{R V B}}\right.$ in this case is $\left.120 \mathrm{~K}\right)$. The former case corresponds to a lower value of the gap $E_{\mathrm{G}}=$ $14 \mathrm{meV}$ even though the characteristic temperature $T_{\mathrm{RVB}}$ is larger, of the order of $210 \mathrm{~K}$. Apart from the highenergy part of the spectrum which drags much too far compared to the experimental cut-off of the order of $50 \mathrm{meV}$, our predictions concerning the value of the spingap and the position of the resonance are in good agreement with the measurements performed in $\mathrm{YBa}_{2} \mathrm{Cu}_{3}$ $\mathrm{O}_{6+x}$ by neutron diffraction. It is believed [54] that self-energy corrections neglected in our mean-field approach would depress the energy tail and restore the correct order of magnitude for the cut-off. More work is required for a proper discussion of the high-energy 


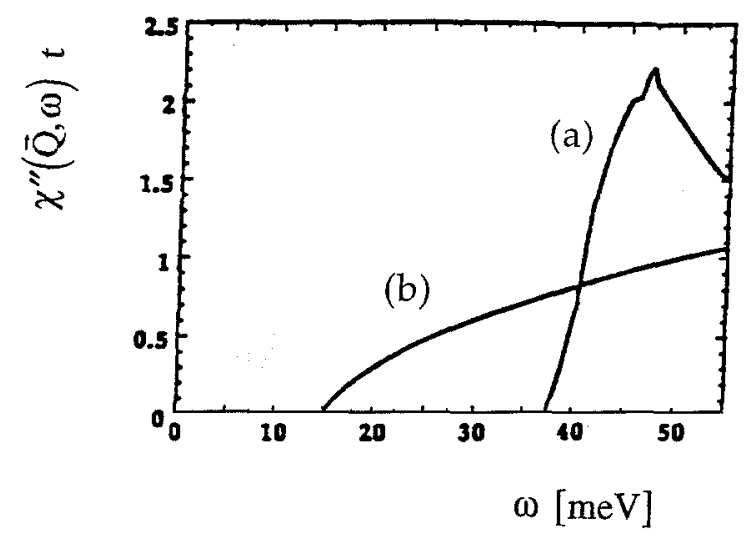

Fig. 5. Frequency dependence of the dynamical susceptibility $\chi(Q, \omega)$ for the same choices of parameters as in Fig. 3. (a) Overdoped case: note the presence of a gap followed by a step and a resonance; (b) heavily doped case.

contribution. Nevertheless, the results at low frequencies show very interesting features that we would like to comment on:

1. The value of the spin-gap $E_{G}$ is found to increase with doping. This result may be surprising at first sight, since, simultaneously, the characteristic pairing temperature $T_{\mathrm{RVB}}$ decreases. Actually, this very atypical behavior results from the interplay between the anisotropy of the Fermi surface and the anisotropy of the pairing parameter $\Delta_{k}$. For instance, it has been shown [40] that in the range of doping such as

$\left\{\mu_{\mathrm{c}_{1}}=4 t^{\prime}+\frac{2 \Delta_{0}}{t^{\prime}}\right\}<\mu<\left\{\mu_{\mathrm{c}_{2}}=\frac{2 \Delta_{0}}{t^{\prime}}\right\}$,

the value of the threshold of antiferromagnetic excitations is

$E_{\mathrm{G}}=8 \Delta_{0} \sqrt{\frac{\mu}{4 t^{\prime}}-\left(\frac{\Delta_{0}}{2 t^{\prime}}\right)^{2}}$.

The last equation, settles the relation between $E_{\mathrm{G}}$ and the pairing parameter $\Delta_{0}$. Due to the anisotropy of the gap parameter $A_{k}$, one can see that $E_{\mathrm{G}}$ scans a whole spectrum of values from $2 \mu$ (insensitive to the effect of $\Delta_{0}$ ) to $2 \sqrt{\left(4 t^{\prime}-\mu\right)^{2}+\left(4 \Delta_{0}\right)^{2}}$ (feeling the full effect of $\Delta_{0}$ ) when $\mu$ goes from $\mu_{\mathrm{c}_{1}}$ to $\mu_{\mathrm{c}_{2}}$. This effect is indeed at the origin of the opposite variation of $E_{\mathrm{G}}$ and $T_{\mathrm{RVB}}$ that we get as a function of doping.

2. The resonance that we obtain at $\omega_{\mathrm{R}}=47 \mathrm{meV}$ has to be brought closer to the superstructure observed by INS in $\mathrm{YBa}_{2} \mathrm{Cu}_{3} \mathrm{O}_{6+x}(x=0.92$ and 1.0). In our scheme, it arises from a dynamical Kohn anomaly of the second kind in the Cooper channel, i.e. proximity of a Van Hove singularity in the density of double excitations

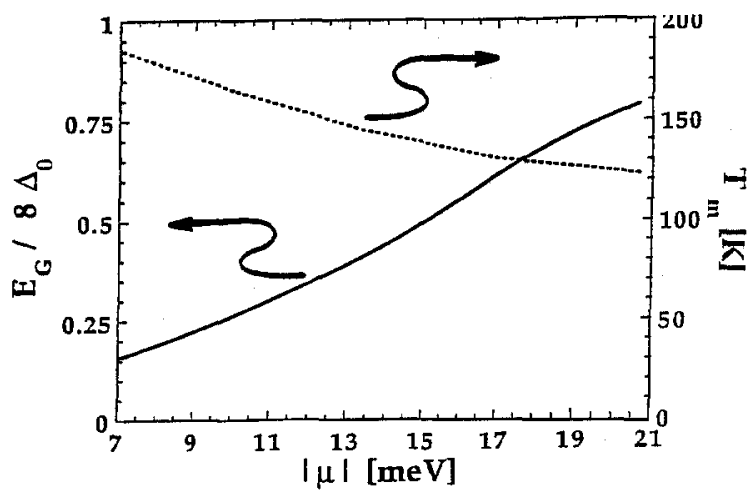

Fig. 6. Combined variation of the spin-gap $E_{\mathrm{G}}$ measured in units $8 \Delta_{0}$, and the temperature $T_{\mathrm{m}}$ at which the spin-gap fills up as a function of the chemical potential.

$\left(\varepsilon_{k}+\varepsilon_{k+Q}\right)$ obtained from pair breaking. The resonance appears as soon as $\mu \geqslant \mu_{c_{1}}$ and progressively vanishes when $\mu$ becomes larger than $\mu_{\mathrm{c}_{2}}$.

Our calculations have also been pursued at finite temperatures. The effect of temperature is to fill up the spin-gap in the regime $4 t^{\prime}<\mu<0$, and to destroy the resonance. The temperature scale $T_{\mathrm{m}}$ at which the spingap opens has been determined from the position of the maximum of $\chi^{\prime \prime}\left(Q, \omega_{0}\right)\left(\omega_{0} \ll E_{\mathrm{G}}\right)$ with temperature. Let us report together the doping dependence that we get for $T_{\mathrm{m}}$ and $E_{\mathrm{G}}$ in Fig. 6 . The apparently contradictory behavior of $E_{\mathrm{G}}$ and $T_{\mathrm{m}}$ observed in neutron diffraction experiments receives a natural explanation in the strongcoupling limit. $T_{\mathrm{m}}$ decreases with doping as $T_{\mathrm{RVB}}$ does, until reaching $T_{\mathrm{m}}=T_{\mathrm{RVB}}=T_{\mathrm{c}}$ at high doping. Meanwhile, $E_{\mathrm{G}}$ increases due to the anisotropy effects mentioned above.

Extending the calculation of $\chi^{\prime \prime}(\boldsymbol{q}, \omega)$ to any value of the momentum $q$, our conclusion is that, for the chosen value of $t^{\prime}, \chi^{\prime \prime}(q, \omega)$ is always peaked around the antiferromagnetic vector $\boldsymbol{Q}=(\pi, \pi)$ with a $\boldsymbol{q}$-widening $\delta \boldsymbol{q}$ which evolves with doping, frequency, and temperature, giving access to the magnetic correlation length $\xi$. We found a characteristic reduction of $\xi$ with doping. It is remarkable that our approach predicts an enhancement of the magnetic correlation length $\xi$ just at the resonance frequency. In our point of view, this gives strong support to our analysis of the resonance as a Kohn anomaly. In the same way, we found that $\xi$ is almost independent of temperature.

\section{Conclusion}

To conclude, we have sketched in this paper the consequences on the spectrum of spin-excitations of a $d_{x^{2}-y^{2}}$ 
pairing in the presence of realistic band-structures (importance of the Fermiology) and strong-coupling effects (role of the proximity of both localization and antiferromagnetic instability) as contained in the $t-t^{\prime}-J$ model. This pairing may as well appear in the superconducting phase, as in the singlet-RVB phase above $T_{\mathrm{c}}$ for the underdoped systems. Our calculatitons show a smooth evolution of the spectrum from one phase to the other, with the existence of a spin-gap in the frequency dependence of $\chi^{\prime \prime}(Q, \omega)$, and a very typical doping dependence of the threshold $E_{\mathrm{G}}$ of antiferromagnetic excitations as compared to the temperature scale $T_{\mathrm{m}}$ associated with the opening of the gap. We were also able to understand the resonance observed for $x=0.92$ or 1.0 as a manifestation of a Kohn anomaly in the Cooper channel. The approach also leads to interesting predictions on NMR quantities which are reported elsewhere [44]. The direction for future work would concern quasi-3D effects with the consideration of interlayer coupling, which seems to play an important role in the underdoped regime. On the other hand, the effects of the gauge field flutuations in the lattice model considered here are likely to be a more delicate problem compared to the continuum limit studied so far [55]. We also consider extending this type of study to heavy-Fermion systems in which a large class of experimental results remains unexplained. This family of systems shares essential features with the superconducting cuprates:

(a) The relevance of strong coupling characterized by the simultaneous proximity to localization via the intrasite Kondo effect, and to a magnetic instability via the intersite RKKY interactions.

(b) The importance of the Fermiology: the effects of the anistropy of the Fermi surfaces as revealed in deHaas-van-Alphen experiments $[56,57]$ for these compounds are largely underestimated in the studies developed so far.

\section{Acknowledgements}

We are grateful to Jean Rossat-Mignod for his constant encouragement and interest in our work. We would like to dedicate this contribution to his memory for both his human and scientific qualities. We would also like to thank L.P. Regnault, P. Burlet, P. Bourges, C. Berthier, M. Horvatic, D. Grempel, K. Fukuyama, K. Maki, S.V. Maleyev, F. Onufrieva, A.M. Tremblay and H. Won for stimulating discussions.

\section{References}

[1] J. Rossat-Mignod, L.P. Regnault, M. J. Jurgens, P. Burlet, J.Y. Henry and G. Lapertot, in: Dynamics of Magnetic
Fluctuations in High Temperature Superconductors, eds. G. Reiter, P. Horsch and G.C. Psaltakis, NATO Advanced Study Institute, Vol. 246 (Plenum Press, New York, 1991) p. 35.

[2] G. Shirane, J. Als-Nielsen, M. Nielsen, J.M. Tranquada, H. Chou, S. Shamoto and M. Sato, Phys. Rev. B 41 (1990) 6547.

[3] J.M. Tranquada, W.J.L. Buyers, H. Chou, T.E. Mason, M. Sato, S. Shamoto and G. Shirane, Phys. Rev. Lett. 64 (1990) 800 .

[4] J. Rossat-Mignod, L.P. Regnault, C. Vettier, P. Burlet, J.Y. Henry and G. Lapertot, Physica B 169 (1991) 58.

[5] J. Rossat-Mignod, L.P. Regnault, C. Vettier, P. Bourges, P. Burlet, J. Bossy, J.Y. Henry and G. Lapertot, Physica C 185-189 (1991) 86.

[6] P.M. Gehring, J.M. Tranquada, G. Shirane, J.R.D. Copley, R.W. Erwin, M. Sato and S. Shamoto, Phys. Rev. B 44 (1991) 2811.

[7] P. Bourges, P.M. Gehring, B. Hennion, A.H. Moudden, I.M. Tranquada, G. Shirane, S. Shamoto and M. Sato, Phys. Rev. B 43 (1991) 8690.

[8] R.J. Birgeneau, R.W. Erwin, P.M. Gehring, M.A. Kastner, B. Keimer, M. Sato, S. Shamoto, G. Shirane and J. Tranquada, Z. Phys. B 87 (1992) 15.

[9] J. Rossat-Mignod, L.P. Regnault, P. Bourges, P. Burlet, C. Vettier and J.Y. Henry, in: Selected Topics in Superconductivity, Vol. 1, eds. L.C. Gupta and M.S. Multani (World Scientific, Singapore, 1993) p. 295.

[10] H.A. Mook, M. Yethiraj, G. Aeppli, T.E. Mason and T. Armstrong, Phys. Rev. Lett. 70 (1993) 3490.

[11] L.P. Regnault, P. Bourges, P. Burlet, J.Y. Henry, J. RossatMignod, Y. Sidis and C. Vettier, Proc. $\mathrm{M}^{2} \mathrm{~S}-\mathrm{HTSC}$ IV, Grenoble, July 1994, to be published in Physica C.

[12] P. Bourges, L.P. Regnault, J.Y. Henry, C. Vettier, Y. Sidis and P. Burlet, Physica B 215 (1995) 127, and references within.

[13] G. Shirane, Physica B 215 (1995) 1.

[14] T.E. Mason, G. Aeppli and H.A. Mook, Phys. Rev. Lett. 68 (1992) 1414.

[15] R.E. Walstedt, W.W. Warren, R. Tycko, R.F. Bell, G.F. Brennert, R.J. Cava, L. Schneemeyer and J. Waszczak, Phys. Rev. B 38 (1989) 9303.

[16] P.C. Hammel, M. Takigawa, R.H. Heffner, Z. Fisk and K.C. Ott, Phys. Rev. Lett. 63 (1989) 1992.

[17] C. Berthier, Y. Berthier, P. Butaud, M. Horvatic, Y. Kitaoka and P. Segransan; in: Dynamics of Magnetic Fluctuations in High Temperature Superconductors, eds. G. Reiter, P. Horsch and G.C. Psaltakis, NATO Advanced Study Institute, Vol. 246 (Plenum Press, New York, 1991) p. 73 .

[18] M. Takigawa, A.P. Reyes, P.C. Hammel, J.D. Tompson, R.H. Heffner, Z. Fisk and K.C. Ott, Phys. Rev. B 43 (1991) 3698 .

[19] H. Alloul, A. Mahajan, H. Casalta and O. Klein, Phys. Rev. Lett. 70 (1993) 1171

[20] M. Horvatic, T. Auler, C. Berthier, Y. Berthier, P. Butaud, W.G. Clark, J.A. Gillet and P. Segransan, Phys. Rev. B 47 (1993) 3461.

[21] M. Takigawa, W. Hults and J.L. Smith, Phys. Rev. Lett. 71 (1993) 2650. 
[22] A. Sokol and D. Pines, Phys. Rev. Lett. 71 (1993) 2813.

[23] C.M. Varma, P.B. Littlewood, S. Schmitt-Rink, E. Abrahams and A.E. Ruckenstein, Phys. Rev. Lett. 63 (1989) 1966.

[24] J.H. Kim, K. Levin and A. Auerbach, Phys. Rev. B 39 (1989) 11633.

[25] Q. Si and K. Levin, Phys. Rev. B 43 (1991) 3075.

[26] Q. Si, Y. Zha, K. Levin and J.P. Lu, Phys. Rev. B 47 (1993) 9055.

[27] N. Bulut and D.J. Scalapino, unpublished.

[28] T. Tanamoto, K. Kuboki and H. Fukuyama, J. Phys. Soc. Japan 60 (1991) 3072.

[29] D.R. Grempel and M. Lavagna, Solid State Commun. 83 (1992) 595.

[30] S.V. Maleyev, J. Phys. 2 (1992) 181.

[31] K. Maki and H. Won, Phys. Rev. Lett. 72 (1994) 1758.

[32] F.C. Zhang and T.M. Rice, Phys. Rev. B 37 (1988) 3759.

[33] J. Campuzano, G. Jennings, M. Faiz, L. Beaulaige, B.W. Veal, J.Z. Linn, A.P. Paulikas, K. Vandervoort, H. Claus, R.S. List, A.J. Arko R.L. Barlett, Phys. Rev. Lett. 64 (1990) 2308.

[34] M.S. Hybertsen, E.B. Stechel, M. Schluter and D.R. Jennison, Phys. Rev. B 41 (1990) 11068.

[35] W. Pickett, Rev. Mod. Phys. 61 (1989) 433

[36] W.E. Pickett, R.E. Cohen and H. Krakauer, Phys. Rev. B 42 (1990) 8764.

[37] H. Chi and A.D.S. Nagi, Phys. Rev. B 43 (1992) 421.

[38] D.M. Newns, P.C. Pattnaik and C.C. Tsuei, Phys. Rev. B 43 (1991) 3075.

[39] P.B. Bénard, L. Chen and A.M.S. Tremblay, Phys. Rev. B 47 (1993) 15217.

[40] M. Lavagna and G. Stemmann, Phys. Rev. B 49 (1994) 4235.
[41] T. Tanamoto, H. Kohno and H. Fukuyama, J. Phys. Soc. Japan 62 (1993) 717.

[42] T. Tanamoto, H. Kohno and H. Fukuyama, J. Phys. Soc. Japan 63 (1994) 2739.

[43] H. Fukuyama, Physica B 215 (1995) 27.

[44] G. Stemmann, C. Pépin and M. Lavagna, Phys. Rev. B 50 (1994) 4075.

[45] G. Stemmann, M. Lavagna and C. Pépin, Proc. $M^{2}$ SHTSC IV, Grenoble, July 1994, to be published in Physica $\mathrm{C}$.

[46] M. Lavagna, G. Stemmann and C. Pépin, J. Low Temp. Phys. 99 (1995) 337.

[47] F. Onufrieva and J. Rossat-Mignod, Proc. M $^{2}$ S-HTSC IV, Grenoble, July 1994, to be published in Physica C.

[48] F. Onufrieva, Physica B 215 (1995) 41.

[49] M. Rice, in: The Physics and Chemistry of Oxide Superconductors, Vol. 60, eds. Y. Iye and H. Yasuoka (Springer, Berlin, 1992) p. 313.

[50] P.W. Anderson, Science 235 (1987) 1196.

[51] G. Kotliar, in: Mechanisms of High Temperature Superconductivity, Vol. 11, eds. H. Kamimura and A. Oshiyama (Springer, Berlin, 1989) p. 61.

[52] N. Nagaosa and P.A. Lee, Phys. Rev. Lett. 64 (1990) 2450.

[53] A. Muramatsu and R. Zeyher, Nucl. Phys. B 346 (1990) 387.

[54] P.B. Littlewood, J. Zaanen, G. Aeppli and H. Monien, Phys. Rev. B 48 (1993) 487.

[55] M. Ubbens and P.A. Lee, Phys. Rev. B 49 (1994) 6853.

[56] L. Taillefer and G.G. Lonzarich, Phys. Rev. Lett. 60 (1988) 1570.

[57] T. Oguchi, A.J. Freeman and G.W. Crabtree, J. Magn. Mater. 63-64 (1987) 645. 